\title{
Geochemistry and tectonic environment of the Dagzhuka ophiolite in the Yarlung-Zangbo suture zone, Tibet
}

\author{
Bin Xia, ${ }^{1,2} *$ Heng-Xiang Yu, ${ }^{1}$ Gen-Wen Chen, ${ }^{1}$ LiAng QI, ${ }^{2,3}$ TAI-Ping ZhaO ${ }^{1}$ and MeI-Fu Zhou ${ }^{3}$ \\ ${ }^{1}$ Guangzhou Institute of Geochemistry, Chinese Academy of Sciences, Guangzhou 510640, China \\ ${ }^{2}$ Institute of Geochemistry, Chinese Academy of Sciences, Guiyang 550002, China \\ ${ }^{3}$ Department of Earth Sciences, University of Hong Kong, Hong Kong
}

(Received January 17, 2001; Accepted December 20, 2002)

\begin{abstract}
The Cretaceous Dagzhuka ophiolite lies in the middle segment of the Yarlung-Zangbo suture zone, Tibet, and has a well-preserved ophiolitic section including a mantle sequence, cumulate complex, diabase dyke swarm and basaltic lavas. Compared to the primitive mantle, tectonized peridotites in the Dagzhuka ophiolite are depleted in $\mathrm{Al}, \mathrm{Ca}$, Ti and high field strength elements, but relatively enriched in large ion lithophile elements ( $\mathrm{Rb}, \mathrm{Ba}, \mathrm{Th})$. They have U-shaped, chondrite-normalized REE patterns suggesting an early stage of depletion by partial melting, followed by enrichment from slab-derived fluids. These features support a model in which the mantle peridotites at Dagzhuka formed in a forearc setting. The diabases and basalts are low-K tholeiites depleted in LREE, with compositions similar to N-MORB. However, these rocks are strongly depleted in $\mathrm{Nb}$ and $\mathrm{Ta}$ but enriched in $\mathrm{Th}$ and $\mathrm{Rb}$, features characteristic of island arc lavas. Thus, the Dagzhuka ophiolite is believed to have formed in a supra-subduction zone environment, possibly a fore-arc basin.
\end{abstract}

\section{INTRODUCTION}

The Yarlung-Zangbo suture zone extends for nearly $1500 \mathrm{~km}$ across southern Tibet and marks the boundary between the Indian and Eurasian Plates (Fig. 1). There are several subparallel tectonic belts, which from North to South include the Gangdese magmatic arc, a continental margin molasse belt, a fore-arc flysch belt, the YarlungZangbo suture itself, a Triassic flysch belt and a band of shelf sedimentary rocks along the northern margin of the Indian Block. Scattered along the suture zone are several ophiolites, which represent relics of Neotethyan oceanic lithosphere (e.g., Nicolas et al., 1981; Allegre et al., 1984; Girardeau et al., 1985; Wang et al., 1987; Zhou et al., 1996; Hao et al., 1999; Wang et al., 1999). The Dagzhuka ophiolite lies in the middle segment of the suture zone and represents an extension of the Xigaze ophiolite to the west. The ophiolite has a well-preserved lithologic sequence ranging from mantle peridotite at the base to pillow lava at the top and thus provides an ideal environment in which to study the evolution of the Tethyan ocean basin (Nicolas et al., 1981; Girardeau et al., 1985; Wang et al., 1987).

Although the ophiolite has been studied extensively, there is no agreement as to its origin or tectonic setting. For example, Girardeau et al. (1985) suggested that it formed at mid-oceanic ridge whereas Wang et al. (1999) favored formation in a small, multi-stage oceanic basin. Here we report the results of extensive field and laboratory studies over the past decade and present a new interpretation of the ophiolite. New major and trace element data place important constraints on the tectonic setting of the Dagzhuka ophiolite and suggest that it formed in a supra-subduction zone environment. 


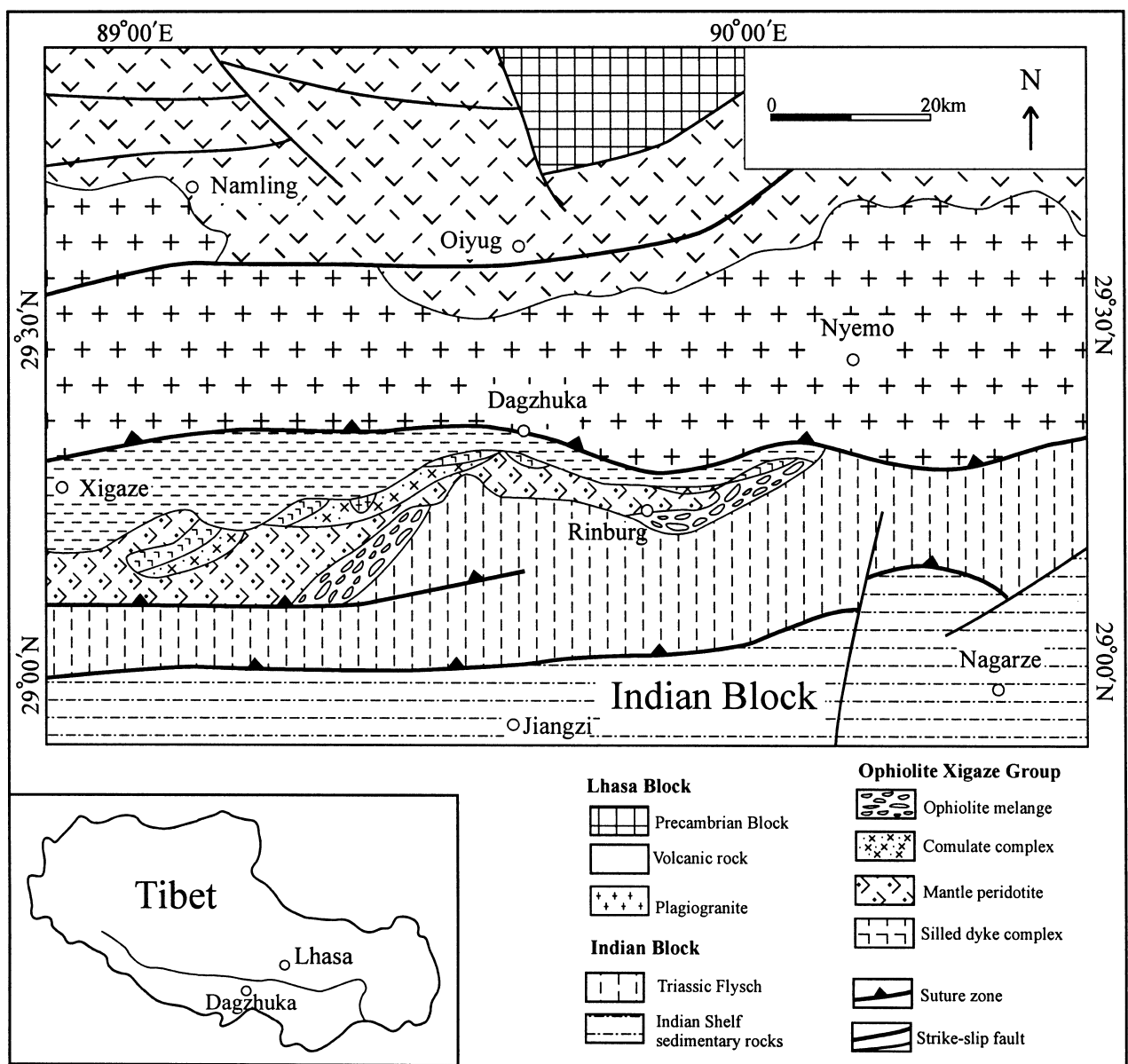

Fig. 1. Geological map of the Dagzhuka area, South Tibet (adapted from Tapponnier et al., 1981; Pearce and Deng, 1988; and XZBGM, 1993).

\section{Geology OF The DAgZhuka OPHiolite}

The Dagzhuka ophiolite is well exposed along the Yarlung-Zangbo River where it forms a section over $2 \mathrm{~km}$ thick (Fig. 2). Although it consists of stacked tectonic slices (Fig. 2), the body is little deformed and contains a nearly complete ophiolite section. The rock units, from the base upward, include mantle peridotite, mafic and ultramafic cumulate rocks, diabase dykes, and pillowed and massive lava flows (Fig. 2). To the south the ophiolite is in fault contact with Triassic flysch deposits and minor Cretaceous mélange. To the north, it is unconformably overlain by the Upper Cretaceous Xigaze Group with a thickness of more than $6000 \mathrm{~m}$. At the contact between the ophiolite and the Xigaze Group there are several meters of radiolian chert (Hao et al., 1999).

The mantle sequence at Dagzhuka has an outcrop width of 10-12 km and consists of mostly fresh rock with only minor serpentinization along fractures. Clinopyroxene-bearing harzburgite and lherzolite with minor dunite pods are the principal rock types. Most of the rocks have crystalloblastic and euhedral-subhedral granular mosaic textures as a result of high-temperature recrystallization.

The cumulate complex overlies the mantle sequence and consists of 500-600 m layered mafic and ultramafic rocks in which three units can be 

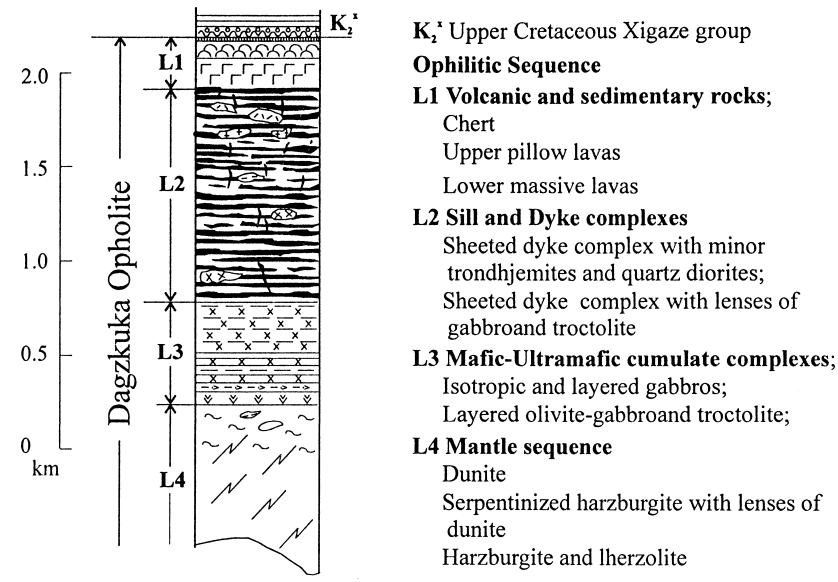

Fig. 2. An idealized column section of the Dagzhuka ophiolite.

recognized. The lower unit is composed dominantly of clinopyroxene-bearing troctolite, which can be distinguished from the mantle peridotite by its well-developed cumulate texture. Near the base of the unit, patches of plagioclase-bearing dunite are locally in contact with the mantle peridotite. The middle unit consists chiefly of layered olivine gabbro, gabbro, and plagioclase-bearing peridotite. The uppermost unit is composed of massive isotropic gabbro and plagiogranite.

A sheeted dyke complex approximately 1000 $m$ wide crops out on the northern side of the ophiolite. The dykes grade upward into pillow lavas with minor sills. In the lower part of the complex dykes intrude plagiogranite, indicating that at least locally they formed later than the cumulate rocks.

The pillowed and massive lavas crop out in a band about 150-200 $\mathrm{m}$ wide between the sheeted dykes and the Upper Cretaceous Xigaze Group (Wang et al., 1987). The lavas are chiefly basalts, with local intercalations of thin-bedded tuffs. A few meters of radiolarian chert occur at the boundary with the Xigaze Group.

\section{Analytical Methods}

We selected only fresh samples for analyses of major and trace elements (including REE). The sample preparation and analyses were carried out at the Institute of Geochemistry, Chinese Academy of Sciences. Major oxides were measured by traditional wet chemical methods, whereas the trace elements of mafic rocks were analyzed by Inductively Coupled Plasma-Mass Spectrometry (ICP-MS) using the techniques of Qi et al. (2000). Samples were diluted for 1000 times.

Because ultramafic rocks have very low trace element concentrations, we developed a special technique to concentrate REEs in solution with only 100 times dilution. Samples of rock powder $(0.25 \mathrm{~g})$ were placed in PTFE crucibles together with $3 \mathrm{ml}$ of $\mathrm{HF}(38 \%)$ and $3 \mathrm{ml}$ of $\mathrm{HNO}_{3}(68 \%)$. The crucibles were then placed on a hot plate, and the solution evaporated to dryness to remove silica. One $\mathrm{ml}$ of $\mathrm{HNO}_{3}$ was then added to each crucible and the solution again evaporated to dryness. At this point, $0.5 \mathrm{ml} \mathrm{HCl}$ and $10 \mathrm{ml} \mathrm{H}_{2} \mathrm{O}$ were added and the crucibles were again heated. After the sample was completely dissolved, $8 \mathrm{ml}$ of $50 \% \mathrm{NaOH}$, previously purified by $\mathrm{Fe}(\mathrm{OH})_{3}$ coprecipitation, and $1 \mathrm{ml}$ of $2 \% \mathrm{MgCl}_{2}$ solution were added, causing the REEs to coprecipitate with $\mathrm{Mg}(\mathrm{OH})_{2}$. The solution was filtered and the precipitate was dissolved in $50 \% \mathrm{HNO}_{3}$. The resulting solution was then transferred to a $25 \mathrm{ml}$ flask and diluted with water to $25 \mathrm{ml}$ for analysis. Because of low concentration of REE in ultramafic rocks, we have doubly purified all chemical agents for sample preparation and cleaned with much 
Table 1. Analytical results (ppm) and detection limit of REEs in reference materials standards (DZE-1, DZE2 and $C A L-S)$

\begin{tabular}{|c|c|c|c|c|c|c|c|}
\hline \multirow[t]{2}{*}{ Element } & \multicolumn{2}{|c|}{ DZE-1 (ultrabasic) } & \multicolumn{2}{|c|}{ DZE-2 (ultrabasic) } & \multicolumn{2}{|c|}{ CAL-S (limestone) } & \multirow[t]{2}{*}{ Detection limits } \\
\hline & Mean (6) & Recommend* & Mean (6) & Recommend* & Mean & Recommend & \\
\hline $\mathrm{Y}$ & 0.064 & & 0.068 & & 2.07 & 2.2 & 0.0091 \\
\hline $\mathrm{La}$ & 0.27 & 0.19 & 0.32 & 0.21 & 0.928 & 0.89 & 0.0062 \\
\hline $\mathrm{Ce}$ & 0.42 & 0.36 & 0.44 & 0.4 & 0.359 & 0.4 & 0.0205 \\
\hline $\operatorname{Pr}$ & 0.035 & & 0.042 & & 0.095 & 0.1 & 0.0062 \\
\hline $\mathrm{Nd}$ & 0.098 & 0.15 & 0.11 & 0.22 & 0.362 & 0.391 & 0.0563 \\
\hline $\mathrm{Sm}$ & 0.018 & 0.02 & 0.021 & 0.03 & 0.069 & 0.071 & 0.0178 \\
\hline $\mathrm{Eu}$ & 0.0037 & 0.004 & 0.0052 & 0.006 & 0.013 & 0.02 & 0.0023 \\
\hline Gd & 0.010 & 0.024 & 0.014 & 0.033 & 0.084 & 0.101 & 0.0012 \\
\hline $\mathrm{Tb}$ & 0.002 & 0.002 & 0.0028 & 0.003 & 0.016 & 0.017 & 0.0003 \\
\hline Dy & 0.012 & & 0.014 & & 0.108 & 0.111 & 0.0014 \\
\hline Ho & 0.0024 & 0.005 & 0.0032 & 0.006 & 0.026 & 0.029 & 0.0005 \\
\hline $\mathrm{Er}$ & 0.0076 & & 0.0090 & & 0.083 & 0.088 & 0.0019 \\
\hline $\mathrm{Tm}$ & 0.0014 & 0.003 & 0.0009 & 0.002 & 0.011 & 0.012 & 0.0001 \\
\hline $\mathrm{Yb}$ & 0.011 & 0.02 & 0.0084 & 0.012 & 0.063 & 0.075 & 0.0010 \\
\hline $\mathrm{Lu}$ & 0.0017 & 0.004 & 0.0012 & 0.003 & 0.009 & 0.011 & 0.0005 \\
\hline
\end{tabular}

*Recommended values of ultramafic rocks, DZE-1 and DZE-2, are from Govindaraju (1994) and are only for references as these data do not yield smooth chondrite-normalized REE patterns. Recommended values of limestone, CAL-S, is from GeoPT6 (International Proficiency Test for Analytical Geochemistry Laboratories, February 2000).

great care the glassware of the instrument. Thus the detection limit is thus much more reduced than the normal samples (Table 1). Recovery of some REE in a limestone (CAL-S) was nearly $100 \%$ (Table 1). There are larger analytical discrepancies between our analyses and reference materials, DZE-1 and DZE-2 (ultramafic rocks) (Table 1). However, the recommended values for these two reference materials were from determination in the 1980's and had large analytical errors (Govindaraju, 1994).

\section{Analytical Results}

\section{Major oxides}

The mantle peridotites are depleted harzburgites with relatively uniform compositions, similar to those in the Troodos, Bay of Islands, and Semail ophiolites (see Coleman, 1977). They have relatively low $\mathrm{Al}_{2} \mathrm{O}_{3}(0.09-1.88 \mathrm{wt} \%)$ and $\mathrm{CaO}(0.20-1.60 \mathrm{wt} \%)$ and high $\mathrm{MgO}$ (37.7 to 42.3 $\mathrm{wt} \%)$, similar to typical mantle harzburgite $(\mathrm{MgO}=39.6-48.4 \mathrm{wt} \%)$. Five of the six analysed samples fall in the field of mantle tectonites on
AFM and ACM diagrams (Fig. 3); the one exception is a dunite (DZ 171) with relatively high $\mathrm{TiO}_{2}$ $(0.12 \mathrm{wt} \%)$. All the other samples are very low in $\mathrm{TiO}_{2}(0.01$ to $0.03 \mathrm{wt} \%$ ) (Table 2).

All of the analysed cumulate rocks (two gabbros and five plagioclase-bearing peridotites and troctolites) have relatively high $\mathrm{Mg \# s}[\mathrm{Mg} /$ (Mg+Fet)] (0.74 and 0.88). In AFM and ACM diagrams (Fig. 3) the samples plot in the field of mafic and ultramafic cumulates and show a tholeiitic trend. As expected, the two gabbros have significantly lower $\mathrm{MgO}(8.40-9.10 \mathrm{wt} \%)$ than the other cumulate rocks (22.4-28.7 wt\%). All of the cumulate rocks are very low in $\mathrm{TiO}_{2}$, ranging from 0.01 to $0.03 \mathrm{wt} \%$ in the peridotites and troctolites and from 0.12 to $0.14 \mathrm{wt} \%$ in the gabbros (Table 2).

The diabases and lavas are basaltic with $\mathrm{SiO}_{2}$ contents ranging from 47.3 to $53.0 \mathrm{wt} \%$ and $\mathrm{TiO}_{2}$ from 0.55 to $0.99 \mathrm{wt} \%$ (with a mean value of 0.82 $\mathrm{wt} \%)$. All of the samples have relatively high $\mathrm{Al}_{2} \mathrm{O}_{3}$ ranging from 16.1 to $19.4 \mathrm{wt} \%$. MgO contents range from 3.20 to $7.50 \mathrm{wt} \%$, and $\mathrm{Mg \# s}$ vary between 0.44 and 0.61 , indicating that these are 


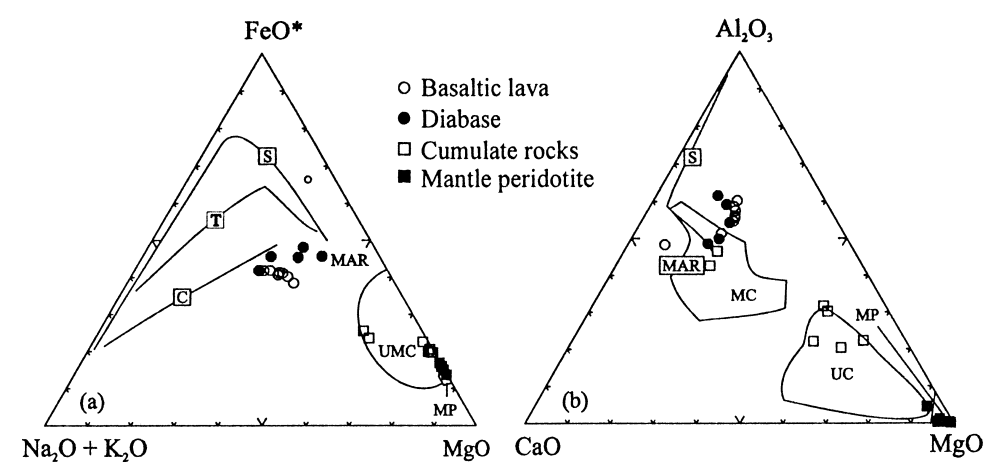

Fig. 3. AFM (a) and ACM (b) diagrams (after Coleman, 1977) for the Dagzhuka ophiolite. C: Cascade calcalkaline trend, T: Thingmuli trend, S: Skaergaard trend, MAR: mid-Atlantic ridge basalts, UMC: ultramaficmafic cumulates, MP: metamorphic peridotite, MC: mafic cumulate, UC: ultramafic cumulate.
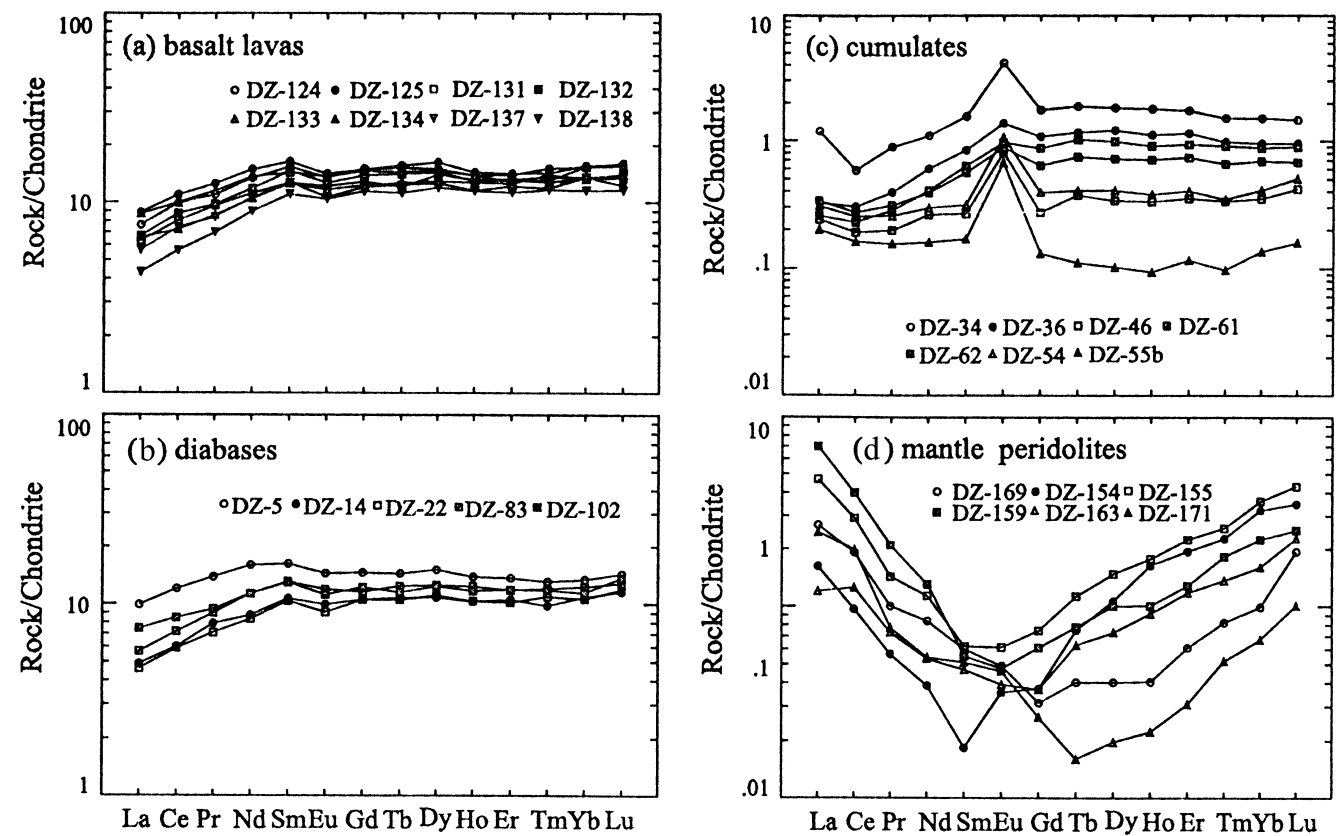

Fig. 4. Chondrite-normalized REE patterns for the Dagzhuka ophiolite. Chondrite values are from Sun (1982).

relatively evolved rocks. Their $\mathrm{K}_{2} \mathrm{O}$ contents are low, between 0.02 and $0.18 \mathrm{wt} \%$.

\section{Rare earth elements (REE)}

The mantle peridotites have pronounced Ushaped chondrite-normalized REE patterns with relative depletion of middle rare earth elements (MREE) (Sm to Tb) and enrichment of both light rare earth elements (LREE) and heavy rare earth elements (HREE) (Fig. 4). (La/Sm) $)_{\mathrm{N}}$ ratios for the six analysed samples range from 2.56 to 12.7 whereas $(\mathrm{Gd} / \mathrm{Yb})_{\mathrm{N}}$ ratios range from 0.12 to 0.40 .

The seven cumulate rock samples have relatively flat, chondrite-normalized REE patterns with slight depletion of LREE and distinct positive Eu anomalies ( $\delta$ Eu ranges from 1.31 to 4.54 ). Total REEs are generally between 0.1 and 2 times chondrite (Table 2, Fig. 4). $(\mathrm{La} / \mathrm{Sm})_{\mathrm{N}}$ and $(\mathrm{Ce} /$ 
Table 2. Major oxides, trace element and rare earth element (REE) abundances for different rock types of the Dagzhuka ophiolite, South Tibet

\begin{tabular}{|c|c|c|c|c|c|c|c|c|}
\hline \multirow{2}{*}{$\begin{array}{l}\text { Rock type } \\
\text { Sample No. }\end{array}$} & \multicolumn{8}{|c|}{ Basalt } \\
\hline & DZ-124 & DZ-125 & DZ-131 & DZ-132 & DZ-133 & DZ-134 & DZ-137 & DZ-138 \\
\hline $\mathrm{SiO}_{2}(\mathrm{wt} \%)$ & 48.2 & 48.6 & 50.0 & 51.7 & 48.4 & 52.1 & 49.9 & 49.3 \\
\hline $\mathrm{TiO}_{2}$ & 0.87 & 0.89 & 0.97 & 0.94 & 0.87 & 0.99 & 0.72 & 0.82 \\
\hline $\mathrm{Al}_{2} \mathrm{O}_{3}$ & 18.4 & 18.7 & 17.5 & 17.61 & 18.0 & 17.7 & 17.7 & 18.7 \\
\hline $\mathrm{Fe}_{2} \mathrm{O}_{3}$ & 3.22 & 3.79 & 2.65 & 3.32 & 1.83 & 2.03 & 1.64 & 3.91 \\
\hline $\mathrm{FeO}$ & 5.38 & 4.81 & 5.85 & 5.08 & 6.17 & 6.27 & 5.56 & 4.49 \\
\hline $\mathrm{MnO}$ & 0.16 & 0.16 & 0.17 & 0.17 & 0.12 & 0.15 & 0.15 & 0.16 \\
\hline $\mathrm{MgO}$ & 6.10 & 6.60 & 6.80 & 6.60 & 3.20 & 5.70 & 6.92 & 7.10 \\
\hline $\mathrm{CaO}$ & 6.90 & 7.20 & 7.70 & 7.20 & 16.00 & 6.00 & 9.80 & 7.80 \\
\hline $\mathrm{Na}_{2} \mathrm{O}$ & 5.29 & 4.87 & 4.87 & 5.10 & 0.73 & 5.50 & 4.17 & 4.75 \\
\hline $\mathrm{K}_{2} \mathrm{O}$ & 0.07 & 0.18 & 0.05 & 0.04 & 0.02 & 0.05 & 0.14 & 0.04 \\
\hline $\mathrm{P}_{2} \mathrm{O}_{5}$ & 0.12 & 0.12 & 0.27 & 0.09 & 0.26 & 0.18 & 0.07 & 0.12 \\
\hline LOI & 4.49 & 3.42 & 2.36 & 1.26 & 3.91 & 2.92 & 2.29 & 2.37 \\
\hline Total & 99.11 & 99.28 & 99.19 & 99.11 & 99.51 & 99.69 & 99.06 & 99.86 \\
\hline $\mathrm{La}(\mathrm{ppm})$ & 2.37 & 2.79 & 2.00 & 2.15 & 2.06 & 2.77 & 1.38 & 1.81 \\
\hline $\mathrm{Ce}$ & 8.11 & 9.08 & 6.73 & 7.39 & 5.96 & 8.33 & 4.71 & 6.21 \\
\hline $\operatorname{Pr}$ & 1.35 & 1.56 & 1.20 & 1.24 & 1.06 & 1.44 & 0.87 & 1.06 \\
\hline $\mathrm{Nd}$ & 7.98 & 8.97 & 6.81 & 7.28 & 6.27 & 8.30 & 5.47 & 6.47 \\
\hline $\mathrm{Sm}$ & 2.95 & 3.18 & 2.49 & 2.89 & 2.44 & 2.80 & 2.17 & 2.49 \\
\hline $\mathrm{Eu}$ & 0.98 & 1.04 & 0.91 & 0.95 & 0.86 & 1.01 & 0.78 & 0.80 \\
\hline Gd & 3.91 & 3.90 & 3.42 & 3.72 & 3.22 & 3.88 & 3.05 & 3.21 \\
\hline $\mathrm{Tb}$ & 0.71 & 0.74 & 0.58 & 0.69 & 0.60 & 0.69 & 0.54 & 0.60 \\
\hline Dy & 4.85 & 5.32 & 4.62 & 4.80 & 4.22 & 4.85 & 3.95 & 4.19 \\
\hline Ho & 1.01 & 1.06 & 0.98 & 0.98 & 0.93 & 1.02 & 0.85 & 0.86 \\
\hline $\mathrm{Er}$ & 2.96 & 3.05 & 2.87 & 2.86 & 2.69 & 2.79 & 2.46 & 2.64 \\
\hline $\mathrm{Tm}$ & 0.50 & 0.48 & 0.46 & 0.44 & 0.47 & 0.44 & 0.40 & 0.41 \\
\hline $\mathrm{Yb}$ & 3.18 & 3.28 & 2.87 & 2.86 & 2.79 & 3.26 & 2.46 & 2.88 \\
\hline $\mathrm{Lu}$ & 0.51 & 0.51 & 0.45 & 0.45 & 0.45 & 0.51 & 0.38 & 0.40 \\
\hline $\mathrm{Sr}$ & 126 & 197 & 117 & 95.2 & 56.8 & 127 & 137 & 61.4 \\
\hline $\mathrm{Rb}$ & 3.22 & 3.45 & 2.48 & 2.25 & 2.38 & 3.52 & 2.50 & 2.12 \\
\hline $\mathrm{Ba}$ & 11.4 & 15.3 & 8.91 & 9.87 & 11.8 & 13.6 & 11.8 & 7.81 \\
\hline Th & 0.34 & 0.28 & 0.25 & 0.26 & 0.32 & 0.46 & 0.23 & 0.25 \\
\hline $\mathrm{Ta}$ & 0.08 & 0.10 & 0.09 & 0.09 & 0.06 & 0.14 & 0.05 & 0.09 \\
\hline $\mathrm{Nb}$ & 1.05 & 1.26 & 1.08 & 1.12 & 0.91 & 1.23 & 0.73 & 0.97 \\
\hline $\mathrm{Zr}$ & 68.9 & 72.7 & 61.3 & 63.3 & 52.6 & 70.8 & 45.9 & 56.8 \\
\hline $\mathrm{Hf}$ & 2.53 & 2.60 & 2.32 & 2.35 & 2.15 & 2.44 & 1.68 & 2.03 \\
\hline Y & 25.0 & 26.6 & 23.1 & 24.3 & 22.4 & 25.4 & 19.9 & 21.8 \\
\hline Sc & 34.1 & 38.6 & 38.6 & 36.6 & 31.6 & 33.8 & 35.9 & 36.4 \\
\hline $\mathrm{Cr}$ & 59.9 & 157 & 116 & 90.6 & 68.5 & 54.8 & 316.4 & 98.1 \\
\hline $\mathrm{Ni}$ & 31.5 & 54.9 & 55.8 & 48.9 & 31.8 & 36.0 & 84.9 & 44.7 \\
\hline V & 245 & 258 & 257 & 253 & 256 & 249 & 225 & 235 \\
\hline Co & 33.3 & 40.2 & 38.3 & 38 & 31.7 & 35.9 & 40.5 & 38.3 \\
\hline $\mathrm{Cu}$ & 42.4 & 34.5 & 58.3 & 52.8 & 11.8 & 25.2 & 68.5 & 46.5 \\
\hline $\mathrm{Zn}$ & 88.1 & 105 & 90.7 & 86.0 & 52.4 & 67.9 & 84.6 & 83.1 \\
\hline $\mathrm{Mg} \#$ & 0.60 & 0.62 & 0.62 & 0.62 & 0.45 & 0.58 & 0.66 & 0.64 \\
\hline$(\mathrm{La} / \mathrm{Sm})_{\mathrm{N}}$ & 0.50 & 0.54 & 0.50 & 0.46 & 0.52 & 0.61 & 0.39 & 0.45 \\
\hline$(\mathrm{Gd} / \mathrm{Yb})_{\mathrm{N}}$ & 0.98 & 0.95 & 0.95 & 1.03 & 0.92 & 0.95 & 0.99 & 0.89 \\
\hline$\Delta \mathrm{eu}$ & 0.89 & 0.91 & 0.95 & 0.90 & 0.94 & 0.94 & 0.93 & 0.87 \\
\hline $\mathrm{Th} / \mathrm{Ta}$ & 4.3 & 2.8 & 2.8 & 2.9 & 5.3 & 3.3 & 4.6 & 2.8 \\
\hline $\mathrm{La} / \mathrm{Ta}$ & 29.6 & 27.9 & 22.2 & 23.9 & 34.4 & 19.8 & 27.7 & 20.1 \\
\hline $\mathrm{Zr} / \mathrm{Nb}$ & 65.6 & 57.7 & 56.8 & 56.5 & 57.8 & 57.5 & 62.9 & 58.5 \\
\hline $\mathrm{Ti} / \mathrm{V}$ & 21.3 & 20.7 & 22.6 & 22.3 & 20.4 & 23.8 & 19.2 & 21.0 \\
\hline
\end{tabular}


Table 2. (continued)

\begin{tabular}{|c|c|c|c|c|c|c|c|c|c|c|}
\hline \multirow{2}{*}{$\begin{array}{l}\text { Rock type } \\
\text { Sample No. }\end{array}$} & \multicolumn{5}{|c|}{ Diabase } & \multicolumn{2}{|c|}{ Gabbro } & \multicolumn{3}{|c|}{ Peridotite } \\
\hline & DZ-5 & DZ-14 & DZ-22 & DZ-83 & DZ-102 & DZ-34 & DZ-36 & DZ-46 & DZ-61 & DZ-62 \\
\hline $\mathrm{SiO}_{2}(\mathrm{wt} \%)$ & 50.2 & 53.0 & 49.9 & 47.3 & 51.0 & 46.8 & 47.9 & 37.7 & 39.0 & 40.9 \\
\hline $\mathrm{TiO}_{2}$ & 0.99 & 0.55 & 0.65 & 0.73 & 0.65 & 0.14 & 0.12 & 0.03 & 0.03 & 0.03 \\
\hline $\mathrm{Al}_{2} \mathrm{O}_{3}$ & 18.00 & 19.4 & 16.1 & 18.4 & 18.0 & 19.6 & 18.7 & 9.5 & 8.7 & 9.5 \\
\hline $\mathrm{Fe}_{2} \mathrm{O}_{3}$ & 5.00 & 2.00 & 2.90 & 3.08 & 3.88 & 0.61 & 0.62 & 2.29 & 4.00 & 4.46 \\
\hline $\mathrm{FeO}$ & 4.79 & 5.22 & 5.20 & 5.62 & 4.32 & 3.29 & 2.59 & 5.01 & 3.47 & 1.94 \\
\hline $\mathrm{MnO}$ & 0.17 & 0.13 & 0.14 & 0.15 & 0.14 & 0.09 & 0.08 & 0.12 & 0.11 & 0.10 \\
\hline $\mathrm{MgO}$ & 6.80 & 4.50 & 6.10 & 7.50 & 5.30 & 9.10 & 8.40 & 28.70 & 27.10 & 23.90 \\
\hline $\mathrm{CaO}$ & 8.30 & 7.60 & 10.90 & 11.00 & 7.10 & 13.4 & 14.6 & 4.20 & 6.90 & 9.30 \\
\hline $\mathrm{Na}_{2} \mathrm{O}$ & 3.09 & 3.69 & 3.22 & 2.40 & 5.46 & 1.76 & 1.35 & 0.14 & 0.23 & 0.24 \\
\hline $\mathrm{K}_{2} \mathrm{O}$ & 0.10 & 0.17 & 0.06 & 0.07 & 0.09 & 0.32 & 0.46 & 0.01 & 0.02 & 0.02 \\
\hline $\mathrm{P}_{2} \mathrm{O}_{5}$ & 1.10 & 1.87 & 3.90 & 2.80 & 2.90 & 0.01 & 0.02 & 0.02 & 0.01 & 0.02 \\
\hline LOI & 1.13 & 1.02 & 0.48 & 0.26 & 0.46 & 4.09 & 4.32 & 11.38 & 9.70 & 8.85 \\
\hline Total & 99.47 & 99.79 & 99.55 & 99.31 & 99.30 & 99.21 & 99.16 & 99.10 & 99.17 & 99.20 \\
\hline $\mathrm{La}(\mathrm{ppm})$ & 3.20 & 1.58 & 1.50 & 1.85 & 2.42 & 0.370 & 0.100 & 0.069 & 0.099 & 0.076 \\
\hline $\mathrm{Ce}$ & 10.23 & 5.09 & 5.04 & 6.14 & 7.24 & 0.476 & 0.247 & 0.144 & 0.210 & 0.178 \\
\hline $\mathrm{Pr}$ & 1.77 & 1.01 & 0.91 & 1.15 & 1.21 & 0.110 & 0.048 & 0.023 & 0.036 & 0.033 \\
\hline $\mathrm{Nd}$ & 9.89 & 5.40 & 5.19 & 7.05 & 7.04 & 0.663 & 0.360 & 0.1460 & 0.221 & 0.232 \\
\hline $\mathrm{Sm}$ & 3.25 & 2.13 & 2.08 & 2.64 & 2.60 & 0.304 & 0.164 & 0.048 & 0.102 & 0.116 \\
\hline $\mathrm{Eu}$ & 1.10 & 0.75 & 0.69 & 0.91 & 0.85 & 0.306 & 0.102 & 0.055 & 0.061 & 0.068 \\
\hline Gd & 3.96 & 2.85 & 2.87 & 3.16 & 3.33 & 0.469 & 0.286 & 0.067 & 0.159 & 0.221 \\
\hline $\mathrm{Tb}$ & 0.71 & 0.53 & 0.52 & 0.61 & 0.57 & 0.090 & 0.056 & 0.016 & 0.034 & 0.046 \\
\hline Dy & 5.10 & 3.63 & 3.77 & 4.26 & 4.20 & 0.604 & 0.397 & 0.102 & 0.223 & 0.310 \\
\hline Ho & 1.05 & 0.78 & 0.79 & 0.94 & 0.89 & 0.137 & 0.082 & 0.023 & 0.049 & 0.064 \\
\hline $\mathrm{Er}$ & 3.03 & 2.32 & 2.27 & 2.63 & 2.64 & 0.374 & 0.245 & 0.069 & 0.149 & 0.191 \\
\hline $\mathrm{Tm}$ & 0.45 & 0.34 & 0.38 & 0.42 & 0.41 & 0.051 & 0.033 & 0.010 & 0.021 & 0.029 \\
\hline $\mathrm{Yb}$ & 2.91 & 2.34 & 2.31 & 2.68 & 2.48 & 0.317 & 0.199 & 0.067 & 0.136 & 0.175 \\
\hline $\mathrm{Lu}$ & 0.48 & 0.39 & 0.40 & 0.43 & 0.46 & 0.047 & 0.031 & 0.012 & 0.021 & 0.027 \\
\hline $\mathrm{Sr}$ & 176 & 171 & 57.9 & 153 & 134 & 188 & 360 & 18.5 & 38.5 & 47.7 \\
\hline $\mathrm{Rb}$ & 2.51 & 2.55 & 2.36 & 2.42 & 3.92 & 6.700 & 6.89 & 2.14 & 2.15 & 2.15 \\
\hline $\mathrm{Ba}$ & 12.9 & 15.5 & 9.84 & 11.0 & 12.1 & 26.2 & 38.0 & 6.50 & 7.02 & 7.18 \\
\hline Th & 0.29 & 0.29 & 0.26 & 0.25 & 0.48 & 0.381 & 0.200 & 0.171 & 0.177 & 0.172 \\
\hline $\mathrm{Ta}$ & 0.10 & 0.06 & 0.07 & 0.12 & 0.13 & 0.192 & 0.099 & 0.032 & 0.032 & 0.036 \\
\hline $\mathrm{Nb}$ & 1.42 & 0.77 & 0.80 & 1.11 & 1.08 & 1.09 & 0.442 & 0.423 & 0.426 & 0.432 \\
\hline $\mathrm{Zr}$ & 89.5 & 51.1 & 50.7 & 45.3 & 60.0 & 4.87 & 3.70 & 2.00 & 2.73 & 2.63 \\
\hline $\mathrm{Hf}$ & 2.78 & 1.98 & 2.00 & 1.90 & 2.22 & 0.673 & 0.231 & 0.104 & 0.171 & 0.173 \\
\hline $\mathrm{Y}$ & 26.6 & 20.0 & 19.5 & 21.8 & 21.8 & 4.63 & 3.84 & 0.721 & 1.33 & 1.74 \\
\hline Sc & 36.6 & 27.3 & 33.2 & 37.6 & 34.5 & 30.2 & 36.8 & 8.59 & 18.0 & 21.2 \\
\hline $\mathrm{Cr}$ & 86.8 & 28.7 & 149 & 325 & 93.5 & 694 & 796 & 2091 & 1614 & 1724 \\
\hline $\mathrm{Ni}$ & 67.8 & 29.4 & 64.4 & 82.5 & 39.9 & 191 & 184 & 1413 & 1122 & 916 \\
\hline V & 278 & 216 & 225 & 223 & 198 & 102 & 125 & 29.6 & 56.9 & 69.5 \\
\hline Co & 40.0 & 28.5 & 34.6 & 40.1 & 32.2 & 30.6 & 29.2 & 106 & 100 & 86.6 \\
\hline $\mathrm{Cu}$ & 57.9 & 11.7 & 27.3 & 67.7 & 35.8 & 32.5 & 128 & 33.6 & 215 & 116 \\
\hline $\mathrm{Zn}$ & 81.5 & 40.8 & 63.1 & 57.3 & 63.3 & 38.8 & 15.6 & 42.0 & 49.1 & 30.5 \\
\hline Mg\# & 0.59 & 0.56 & 0.61 & 0.64 & 0.58 & 0.83 & 0.84 & 0.89 & 0.88 & 0.89 \\
\hline$(\mathrm{La} / \mathrm{Sm})_{\mathrm{N}}$ & 0.61 & 0.46 & 0.45 & 0.43 & 0.57 & 0.75 & 0.38 & 0.90 & 0.60 & 0.40 \\
\hline$(\mathrm{Gd} / \mathrm{Yb})_{\mathrm{N}}$ & 1.09 & 0.97 & 0.99 & 0.94 & 1.07 & 1.18 & 1.15 & 0.79 & 0.93 & 1.01 \\
\hline$\Delta \mathrm{eu}$ & 0.94 & 0.94 & 0.87 & 0.97 & 0.89 & 2.49 & 1.45 & 3.02 & 1.48 & 1.31 \\
\hline $\mathrm{Th} / \mathrm{Ta}$ & 2.9 & 4.8 & 3.7 & 2.1 & 3.7 & 2.0 & 2.0 & 5.3 & 5.5 & 4.8 \\
\hline $\mathrm{La} / \mathrm{Ta}$ & 32.0 & 26.3 & 21.5 & 15.4 & 18.6 & 1.9 & 1.0 & 2.2 & 3.1 & 2.1 \\
\hline $\mathrm{Zr} / \mathrm{Nb}$ & 63.0 & 66.4 & 63.4 & 40.8 & 55.5 & 4.5 & 8.4 & 4.7 & 6.4 & 6.1 \\
\hline $\mathrm{Ti} / \mathrm{V}$ & 21.4 & 15.3 & 17.3 & 19.6 & 19.7 & 8.2 & 5.7 & 6.1 & 3.2 & 2.6 \\
\hline
\end{tabular}


Table 2. (continued)

\begin{tabular}{|c|c|c|c|c|c|c|c|c|}
\hline \multirow{2}{*}{$\begin{array}{l}\text { Rock type } \\
\text { Sample No. }\end{array}$} & \multicolumn{2}{|c|}{ Troctolite } & \multicolumn{5}{|c|}{ Harzburgite } & \multirow{2}{*}{$\begin{array}{l}\text { Dunite } \\
\text { DZ-171 }\end{array}$} \\
\hline & DZ-54 & DZ-55b & DZ-169 & DZ-154 & DZ-155 & DZ-159 & DZ-163 & \\
\hline $\mathrm{SiO}_{2}(\mathrm{wt} \%)$ & 37.8 & 38.9 & 39.1 & 41.9 & 42.8 & 41.2 & 41.1 & 37.8 \\
\hline $\mathrm{TiO}_{2}$ & 0.03 & 0.01 & 0.02 & 0.01 & 0.02 & 0.02 & 0.02 & 0.12 \\
\hline $\mathrm{Al}_{2} \mathrm{O}_{3}$ & 13.5 & 13.2 & 0.27 & 1.88 & 0.47 & 0.18 & 0.18 & 0.09 \\
\hline $\mathrm{Fe}_{2} \mathrm{O}_{3}$ & 3.86 & 3.16 & 1.00 & 2.15 & 1.24 & 1.83 & 1.36 & 2.10 \\
\hline $\mathrm{FeO}$ & 3.84 & 2.84 & 6.82 & 5.55 & 5.56 & 6.27 & 6.24 & 4.80 \\
\hline $\mathrm{MnO}$ & 0.12 & 0.09 & 0.12 & 0.13 & 0.13 & 0.14 & 0.13 & 0.10 \\
\hline $\mathrm{MgO}$ & 24.60 & 22.40 & 42.00 & 37.80 & 37.70 & 38.90 & 39.30 & 42.30 \\
\hline $\mathrm{CaO}$ & 6.40 & 6.10 & 0.40 & 1.60 & 1.00 & 1.40 & 0.90 & 0.20 \\
\hline $\mathrm{Na}_{2} \mathrm{O}$ & 0.39 & 0.35 & 0.08 & 0.09 & 0.09 & 0.10 & 0.11 & 0.09 \\
\hline $\mathrm{K}_{2} \mathrm{O}$ & 0.05 & 0.04 & 0.02 & 0.02 & 0.02 & 0.01 & 0.01 & 0.02 \\
\hline $\mathrm{P}_{2} \mathrm{O}_{5}$ & 0.01 & 0.02 & 0.20 & 0.03 & 0.03 & 0.18 & 0.18 & 0.07 \\
\hline LOI & 8.93 & 12.01 & 9.43 & 8.17 & 9.86 & 9.20 & 9.92 & 11.70 \\
\hline Total & 99.54 & 99.12 & 99.36 & 99.63 & 99.12 & 99.15 & 99.45 & 99.39 \\
\hline $\mathrm{La}(\mathrm{ppm})$ & 0.091 & 0.057 & 0.079 & 0.049 & 0.138 & 0.208 & 0.035 & 0.071 \\
\hline $\mathrm{Ce}$ & 0.197 & 0.121 & 0.149 & 0.076 & 0.225 & 0.310 & 0.097 & 0.151 \\
\hline $\operatorname{Pr}$ & 0.030 & 0.018 & 0.012 & 0.007 & 0.017 & 0.025 & 0.0085 & 0.0088 \\
\hline $\mathrm{Nd}$ & 0.169 & 0.088 & 0.048 & 0.022 & 0.064 & 0.074 & 0.030 & 0.030 \\
\hline Sm & 0.058 & 0.030 & 0.011 & 0.003 & 0.011 & 0.010 & 0.0085 & 0.0091 \\
\hline $\mathrm{Eu}$ & 0.075 & 0.045 & 0.003 & 0.003 & 0.004 & 0.003 & 0.003 & 0.003 \\
\hline Gd & 0.099 & 0.032 & 0.0078 & 0.0094 & 0.018 & 0.015 & 0.0091 & 0.0064 \\
\hline $\mathrm{Tb}$ & 0.018 & 0.0048 & 0.0018 & 0.0034 & 0.0050 & 0.0035 & 0.0028 & 0.0007 \\
\hline Dy & 0.128 & 0.031 & 0.012 & 0.033 & 0.045 & 0.031 & 0.022 & 0.0059 \\
\hline Ho & 0.027 & 0.0063 & 0.003 & 0.012 & 0.012 & 0.007 & 0.006 & 0.0015 \\
\hline $\mathrm{Er}$ & 0.082 & 0.023 & 0.012 & 0.040 & 0.045 & 0.026 & 0.024 & 0.0061 \\
\hline $\mathrm{Tm}$ & 0.011 & 0.003 & 0.0026 & 0.0073 & 0.0081 & 0.0058 & 0.0043 & 0.0016 \\
\hline $\mathrm{Yb}$ & 0.081 & 0.026 & 0.020 & 0.064 & 0.070 & 0.045 & 0.032 & 0.013 \\
\hline $\mathrm{Lu}$ & 0.016 & 0.005 & 0.006 & 0.0107 & 0.0130 & 0.0077 & 0.0069 & 0.0030 \\
\hline $\mathrm{Sr}$ & 71.0 & 104.3 & 11.2 & 11.2 & 12.7 & 11.9 & 11.3 & 11.2 \\
\hline $\mathrm{Rb}$ & 2.15 & 2.38 & 2.06 & 2.02 & 2.11 & 2.09 & 2.04 & 2.04 \\
\hline $\mathrm{Ba}$ & 6.55 & 14.3 & 6.75 & 5.95 & 6.96 & 7.24 & 6.89 & 6.44 \\
\hline Th & 0.168 & 0.171 & 0.182 & 0.166 & 0.238 & 0.191 & 0.182 & 0.176 \\
\hline $\mathrm{Ta}$ & 0.031 & 0.031 & 0.036 & 0.033 & 0.049 & 0.038 & 0.035 & 0.031 \\
\hline $\mathrm{Nb}$ & 0.456 & 0.418 & 0.444 & 0.420 & 0.440 & 0.436 & 0.419 & 0.439 \\
\hline $\mathrm{Zr}$ & 2.20 & 2.48 & 1.95 & 1.67 & 1.72 & 1.71 & 1.82 & 1.85 \\
\hline $\mathrm{Hf}$ & 0.107 & 0.136 & 0.102 & 0.098 & 0.096 & 0.102 & 0.094 & 0.098 \\
\hline $\mathrm{Y}$ & 0.703 & 0.236 & 0.086 & 0.243 & 0.300 & 0.176 & 0.154 & 0.046 \\
\hline $\mathrm{Sc}$ & 9.19 & 4.42 & 7.73 & 13.9 & 13.1 & 14.8 & 11.3 & 4.88 \\
\hline $\mathrm{Cr}$ & 2041 & 3145 & 2835 & 2657 & 2290 & 3089 & 3133 & 2941 \\
\hline $\mathrm{Ni}$ & 1051 & 1016 & 2391 & 2286 & 2102 & 2275 & 2366 & 2826 \\
\hline V & 34.2 & 24.6 & 23.2 & 57.22 & 52.40 & 56.20 & 42.17 & 11.12 \\
\hline Co & 90.9 & 84.1 & 132 & 123 & 124 & 125 & 125 & 139 \\
\hline $\mathrm{Cu}$ & 92.6 & 84.0 & 4.93 & 20.7 & 13.1 & 29.2 & 5.63 & 2.96 \\
\hline $\mathrm{Zn}$ & 42.7 & 37.1 & 42.4 & 42.6 & 41.3 & 48.3 & 44.8 & 37.0 \\
\hline Mg\# & 0.92 & 0.91 & 0.92 & 0.91 & 0.92 & 0.91 & 0.91 & 0.93 \\
\hline$(\mathrm{La} / \mathrm{Sm})_{\mathrm{N}}$ & 0.97 & 1.17 & 4.48 & 8.80 & 7.59 & 12.71 & 2.56 & 4.79 \\
\hline$(\mathrm{Gd} / \mathrm{Yb})_{\mathrm{N}}$ & 0.97 & 0.97 & 0.32 & 0.12 & 0.21 & 0.27 & 0.23 & 0.40 \\
\hline$\Delta \mathrm{eu}$ & 3.07 & 4.54 & 1.13 & 1.36 & 0.90 & 0.82 & 0.94 & 1.25 \\
\hline $\mathrm{Th} / \mathrm{Ta}$ & 5.4 & 5.5 & 5.1 & 5.0 & 4.9 & 5.0 & 5.2 & 5.7 \\
\hline $\mathrm{La} / \mathrm{Ta}$ & 2.9 & 1.8 & 2.2 & 1.5 & 2.8 & 5.5 & 1.0 & 2.3 \\
\hline $\mathrm{Zr} / \mathrm{Nb}$ & 4.8 & 5.9 & 4.4 & 4.0 & 3.9 & 3.9 & 4.3 & 4.2 \\
\hline $\mathrm{Ti} / \mathrm{V}$ & 5.3 & 2.4 & 5.2 & 1.0 & 2.3 & 2.1 & 2.8 & 64.7 \\
\hline
\end{tabular}

Notes: $\mathrm{Mg \#}$ is mole fractionation of $\mathrm{Mg}^{+2} /\left(\mathrm{Mg}^{+2}+\mathrm{Fe}_{t}^{+2}\right) ;(\mathrm{La} / \mathrm{Sm})_{N}$ is chondrite-normalized ratio of La/Sm. Chondrite values are from Sun (1982). 

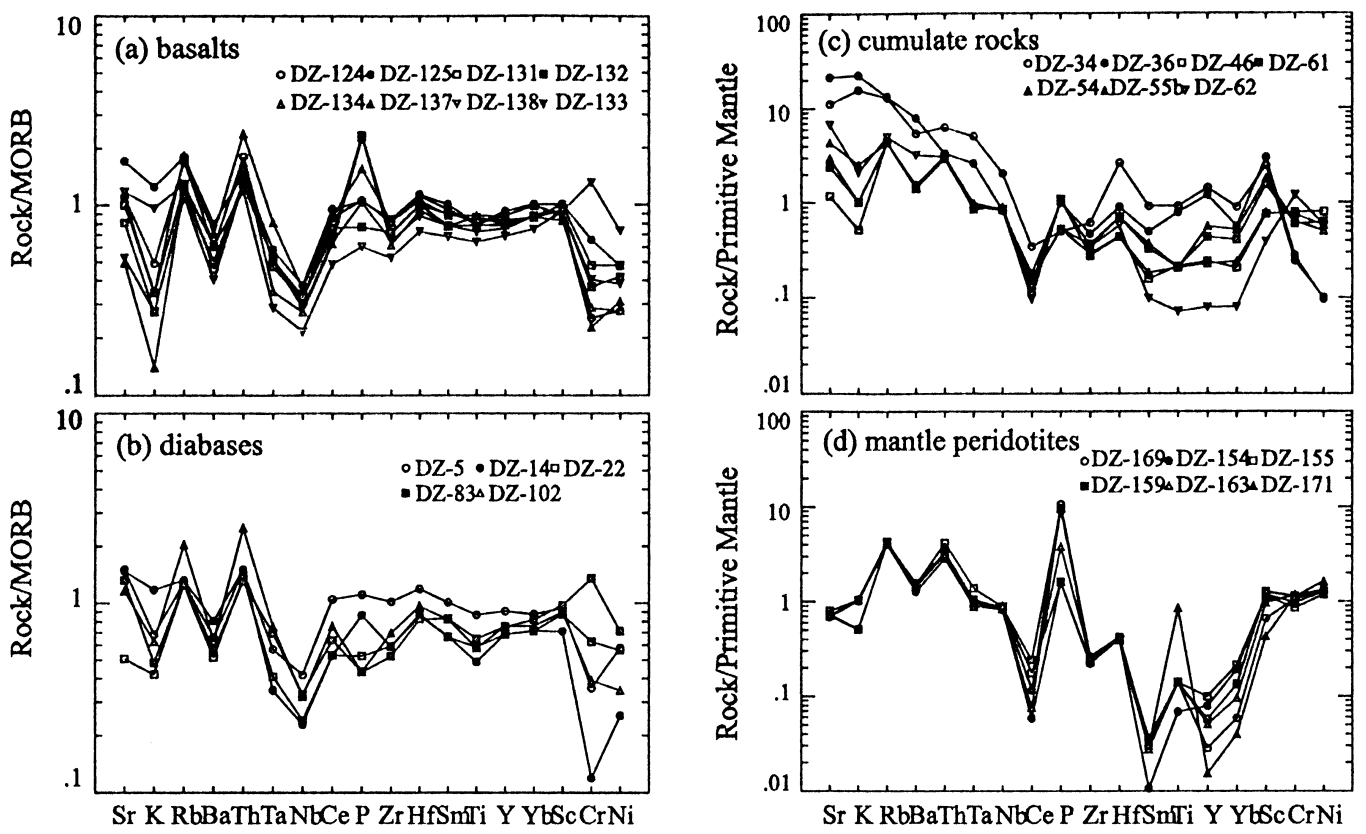

Fig. 5. MORB and primitive mantle-normalized trace element spider diagrams for the Dagzhuka ophiolite. MORB and primitive mantle normalization values are from Pearce (1982) and Taylor and McLennan (1985), respectively.

$\mathrm{Yb})_{\mathrm{N}}$ ratios are less than those of chondrite and primitive mantle.

The diabases and lavas have uniform, flat chondrite-normalized REE patterns with moderate light REE depletion. $(\mathrm{La} / \mathrm{Sm})_{\mathrm{N}}$ ratios are between 0.39 and $0.61,(\mathrm{La} / \mathrm{Yb})_{\mathrm{N}}$ between 0.38 and 0.74 , and $(\mathrm{Ce} / \mathrm{Sm})_{\mathrm{N}}$ between 0.51 and 0.74 . All the samples show a weak negative Eu anomaly, with $\delta$ Eu values ranging from 0.87 to 0.97 (Fig. 4 , Table 2). These REE patterns are similar to those of N-MORB but are also comparable to those of pillow lavas in the Troodos, Semail and Bay of Island ophiolites (Suen et al., 1979; Alabaster et al., 1982; Cameron, 1985; Thy and Moores, 1988; Jenner et al., 1991; Elthon, 1991).

\section{Trace elements}

Relative to the primitive mantle, the peridotites are enriched in $\mathrm{Rb}, \mathrm{Ba}, \mathrm{U}, \mathrm{Th}, \mathrm{P}$ and other large ion lithophile elements (LILE), but depleted in Ce, $\mathrm{Zr}, \mathrm{Hf}, \mathrm{Sm}, \mathrm{Ti}, \mathrm{Y}, \mathrm{Yb}$ and other high field strength elements (HFSE) (Fig. 5). The cumulate rocks have somewhat similar trace element composi- tions, being enriched in $\mathrm{Sr}, \mathrm{K}, \mathrm{Rb}, \mathrm{Ba}, \mathrm{U}$, Th and other LILEs, but depleted in $\mathrm{Ce}, \mathrm{Zr}, \mathrm{Hf}, \mathrm{Sm}$, Ti, $\mathrm{Y}, \mathrm{Yb}$ and other HFSE. The cumulate rocks are less depleted in HFSE than the mantle peridotites but more enriched in LILE.

Diabases and lavas have similar trace element characteristics and the contents of HFSE such as $\mathrm{Ce}, \mathrm{Zr}, \mathrm{Hf}, \mathrm{Sm}, \mathrm{Y}, \mathrm{Yb}$ and $\mathrm{Sc}$ are close to those of MORB (Pearce, 1982). On MORB-normalized spider diagrams the samples are enriched in $\mathrm{Rb}$ and $\mathrm{Th}$ but depleted in $\mathrm{K}, \mathrm{Ba}, \mathrm{Nb}$ and $\mathrm{Ta}$ (Fig. 5).

\section{DISCUSSION}

Ophiolites in the Yarlung-Zangbo suture zone were formed in the Early Cretaceous about 120$130 \mathrm{Ma}$ ) (Marcoux et al., 1982; Gopel et al., 1984; Wu, 1984; Wang et al., 1987), and were nearly contemporaneous with the early plutonic and volcanic rocks of the Gangdese arc (about 110-120 Ma) (XZBGM, 1993). The Dagzhuka ophiolite is overlain comformably by forearc sedimentary rocks of the Late Cretaceous (Cenomanian) Xigaze 

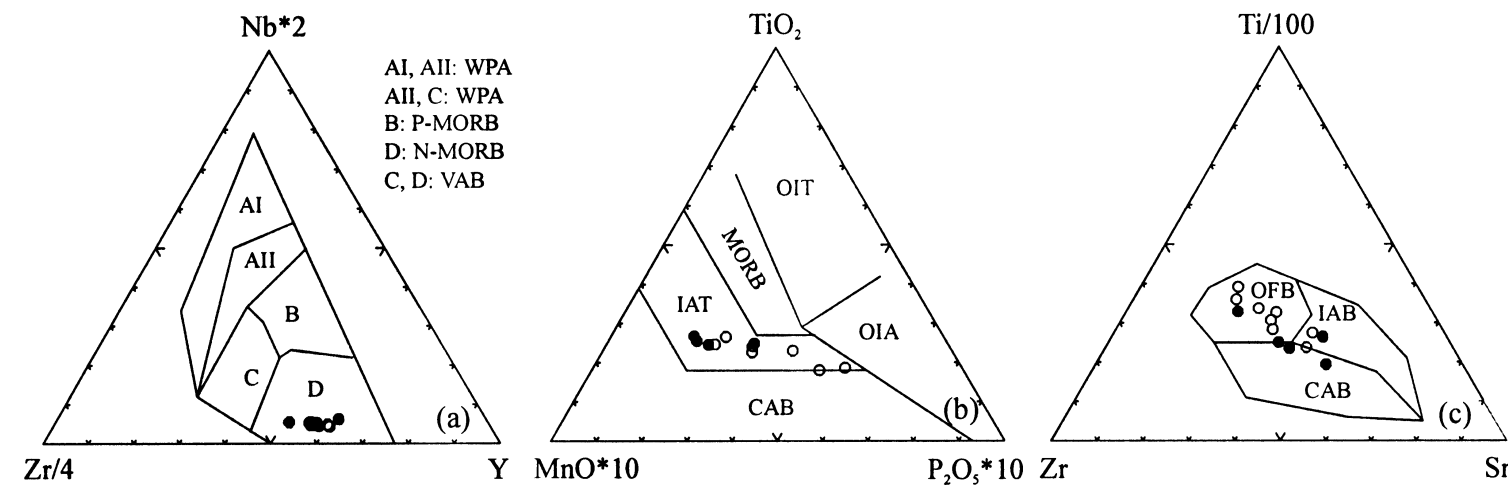

$\mathrm{MnO}^{*} 10$

$\mathrm{P}_{2} \mathrm{O}_{5} * 10 \mathrm{Zr}$

Fig. 6. Geochemistry and tectonic environment diagrams of the Dagzhuka ophiolite. The samples are basalt (open circles) and diabases (filled circles) from Table 2. (a) Nb*2-Zr/4-Y diagram after Meschede (1986). (b) $\mathrm{TiO}_{2}-\mathrm{MnO}-\mathrm{P}_{2} \mathrm{O}_{5}$ diagram after Mullen (1983). IAT: island arc tholeiite; MORB: mid-ocean ridge basalt; OIA: ocean island alkaline basalt; OIT: ocean island tholeiite; CAB: calc-alkaline basalt. (c) Ti/100-Zr-Sr/2 diagram after Pearce and Cann (1973). OFB: ocean floor basalt (MORB); IAB: island arc basalt; CAB: calcalkaline basalt.

Group (XZBGM, 1993; Durr, 1996; Hao et al., 1999). The Xigaze Group consists of flysch sediments deposited on a continental slope or in the Gangdese forearc basin (Wang et al., 1987; XZBGM, 1993; Durr, 1996; Hao et al., 1999; Wang et al., 1999). Both subduction-related melange and blueschists occur on the southern side of the mantle peridotite in the lower part of the ophiolite (Wang et al., 1987).

The lithologic section and major oxides of the rocks are similar in some respects to those formed at a mid-ocean ridge, although the section is only half as thick as typical oceanic crust. The small thickness $(<600 \mathrm{~m})$ of the cumulate sequence (Girardeau et al., 1985; Wang et al., 1987) suggests a low magma supply and possibly a slow spreading rate (Girardeau et al., 1985; Girardeau and Mercier, 1988; Wang et al., 1987). On the other hand, the trace element geochemistry and regional geology suggest that the Dagzhuka ophiolite was formed in a suprasubduction zone environment (Figs. 5 and 6), possibly in the Gangdese fore-arc basin. Spreading in the Gangdese fore-arc in the Early Cretaceous is consistent with coeval back-arc rifting in southern Tibet as documented by Zhang (2000).

Pearce et al. (1984) classified ophiolites as mid-ocean ridge types (MOR-type) and supra-sub- duction zone types (SSZ-type). According to these authors, SSZ-type ophiolites can form in the upper plate during the early stages of subduction. SSZ-type ophiolites can be developed either in a forearc or back-arc environment, but the presence of boninites in many ophiolites suggests formation in forearc environments. Such ophiolites are characterized by thin crustal sections and by the presence of island-arc and fore-arc lavas.

Mantle peridotites in SSZ-type ophiolites are strongly depleted in HFSE, such as Ti. The mantle peridotites of the Dagzhuka ophiolite are depleted in Al, Ca, REE (especially MREE) and HFSE (Zr, Hf, Ti, Y) but relatively enriched in LILE (Rb, Ba, U, Th). These features suggest formation of the Dagzhuka ophiolite in a SSZ environment. Because the rocks are fresh the LILE enrichment cannot be related to serpentinization and is best explained by metasomatism of the mantle by fluids released from a subducted slab (Gruau et al., 1998). The distinctive U-shaped chondrite-normalized REE patterns of the peridotites can be explained by a two-stage process: an early stage of depletion due to partial melting, followed by selective enrichment of LILE related to mantle metasomatism (cf., Zhou et al., 2000, 2001). Therefore, it is possible that the mantle peridotites in Dagzhuka formed in a SSZ envi- 


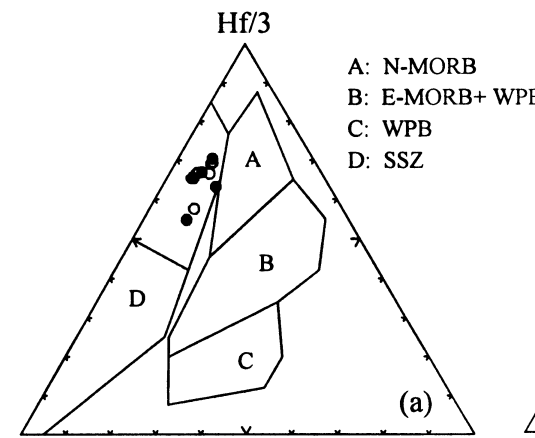

$\mathrm{Th}$

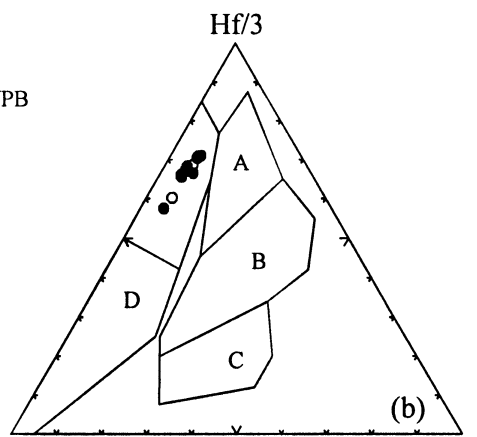

$\mathrm{Nb} / 16 \mathrm{Th}$

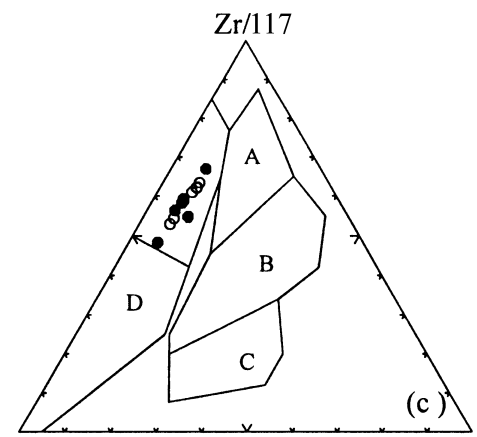

$\mathrm{Nb} / 16$

Fig. 7. (a) Hf/3-Th-Ta, (b) Hf/3-Th-Nb/16, and (c) Zr/117-Th-Nb/16 diagrams after Wood (1980). $N$-MORB: normal type mid-ocean ridge basalt; E-MORB: enriched type mid-ocean ridge basalt; WPB: withinplate basalt; SSZ: supra-subduction zone, Back-arc and fore-arc basalts also plot within the SSZ field.
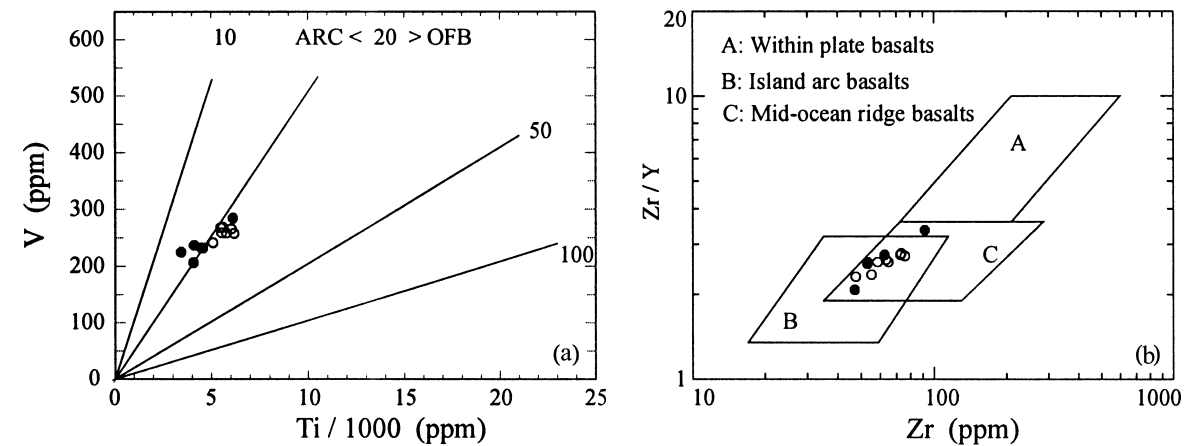

Fig. 8. (a) Ti/1000-V diagram after Shervais (1982). ARC: arc basalt; OFB: ocean floor basalt (MORB). (b) Zr$\mathrm{Zr} / \mathrm{Y}$ diagram after Pearce and Norry (1979).

ronment.

Major oxides of the diabases and lavas in Dagzhuka are similar to mafic volcanic rocks in the Troodos, Semail, and Bay of Islands ophiolites, and to N-MORB in the ocean basins (Pearce, 1975; Sun et al., 1979; Alabaster et al., 1982; Cameron, 1985; Thy et al., 1988), but with somewhat higher $\mathrm{Al}_{2} \mathrm{O}_{3}$. These rocks show marked depletion in $\mathrm{Nb}$ and Ta but enrichment in Th and $\mathrm{Rb}$, features considered typical of SSZ environments. On $\mathrm{Zr} / 4$ $\mathrm{Yb}^{*} 2-\mathrm{Y}, \mathrm{MnO}-\mathrm{TiO}_{2}-\mathrm{P}_{2} \mathrm{O}_{5}$, Th-Hf/3-Ta, Th-Hf/3$\mathrm{Nb} / 16$, and $\mathrm{Th}-\mathrm{Zr} / 117-\mathrm{Nb} / 16$ discrimination diagrams all the diabases and lavas in Dagzhuka plot within the SSZ fields (Figs. 6 and 7). However, on Ti/100-Zr-Sr/2, Ti/1000-V and Zr/Y-Zr diagrams (Fig. 8), they plot within the overlapping fields of island-arc basalts and mid-ocean ridge basalts. The $\mathrm{Th} / \mathrm{Ta}$ and $\mathrm{La} / \mathrm{Ta}$ ratios of the diabases and basaltic lavas (2.1-5.3 and 15.4-34.4, respectively; Table 2), are similar to those of ophiolitic lavas formed in SSZ environment $(\mathrm{Th} / \mathrm{Ta}=3-5$, $\mathrm{La} / \mathrm{Ta}=30-40)$ but higher than those of MORB $(\mathrm{Th} / \mathrm{Ta}=0.75-2, \mathrm{La} / \mathrm{Ta}=10-20)$ (Elthon, 1991; Pearce, 1991). The REE distribution patterns of the diabases and basaltic lavas are similar to those of the cumulate rocks, implying that they were comagmatic. The pronounced $\mathrm{Eu}$ anomalies in the cumulate rocks reflect plagioclase accumulation.

Although we did not identify in this study boninitic rocks in the crustal sequence in Dagzhuka, boninites were reported elsewhere in the Xigaze ophiolite to the west (Wang et al., 


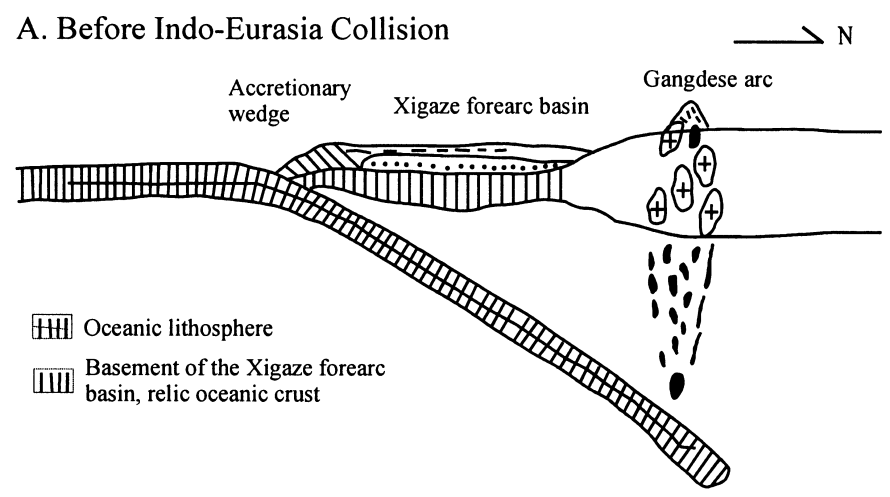

B. After Indo-Asian Collision

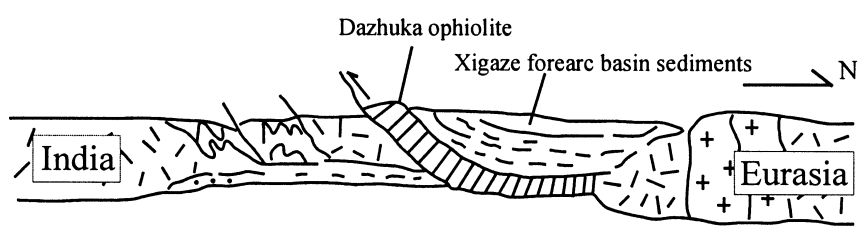

Fig. 9. Diagram showing our interpretation of the formation and emplacement of the Dagzhuka ophiolite.

1987). The Luobusa ophiolite to the east contains high-Cr podiform chromite deposits (Zhou et al., 1996). Small pods of chromitites were also discovered in the mantle sequence of the Dagzhuka ophiolite (Wang et al., 1987). These high-Cr chromite bodies are indicative of formation from boninitic magmas in a SSZ environment (Zhou et al., 2000, 2001). Thus, both the mantle peridotites and crustal rocks have compositions suggesting formation in a SSZ environment. We suggest that the Dagzhuka ophiolite was formed in the Gangdese forearc basin that developed in response to subduction of Tethys during the Cretaceous (Fig. 9). This model contrasts previous mid-oceanic ridge environment of the ophiolite (Girardeau et al., 1985).

\section{Conclusions}

The principal conclusions are:

(1) The Dagzhuka ophiolite has a mantle sequence depleted in Al, Ca, REE (especially MREE), HFSE (Zr, Hf, Ti, Y), but relatively en- riched in LILE (Rb, Ba, U, Th), features typical of SSZ-type ophiolites.

(2) The diabases and lavas in the Dagzhuka ophiolite are low-K, high-Al basalts. They have flat, LREE-depleted REE patterns but their LILE distributions differ markedly from MORB. In particular, the rocks are strongly depleted in $\mathrm{Nb}$ and $\mathrm{Ta}$, relatively depleted in $\mathrm{K}$ and $\mathrm{Ba}$ and strongly enriched in $\mathrm{Th}$ and $\mathrm{Rb}$, and are typical island-arc lavas.

(3) The Dagzhuka ophiolite formed in a SSZ environment, possibly in a forearc environment in response to subduction of the Tethys during the Early Cretaceous.

Acknowledgments-The work was supported jointly by the National Natural Science Foundation of China (No. 40072022), the Chinese Academy of Sciences GRANT (No. KZCXZ-SW-117), the National Climbing Program of China (95-Yu-25-03), the National Key Basic Research Program of China (G1999043204), the Director's Foundation (CAS) and the Foundation of the Laboratory of Ore Deposit Geochemistry (CAS). Xiang-Lin Tu and Sun-Rong Li helped with sample 
analyses. We thank Profs. Guo-Qin Zhou, Yi-Gang Xu, Xian-Hua $\mathrm{Li}$ and Ji-Feng $\mathrm{Xu}$ for helpful discussion. Journal reviews by Dr. T. Ahmad helped improve an earlier version and are gratefully acknowledged.

\section{REFERENCES}

Alabaster, T., Pearce, J. A. and Malpas, J. (1982) The volcanic stratigraphy and petrogenesis of the Oman ophiolite complex. Contrib. Mineral. Petrol. 81, 168183.

Allegre, C. J. and 34 others (1984) Structure and evolution of the Himalaya-Tibet orogenic belt. Nature 307, 17-22.

Cameron, W. E. (1985) Petrology and origin of primitive lavas from the Troodos ophiolite, Cyprus. Contrib. Mineral. Petrol. 89, 239-255.

Coleman, R. G. (1977) Ophiolites: Ancient Oceanic Lithosphere. Springer-Verlag, New York, 229 pp.

Durr, S. B. (1996) Provenance of Xigaze fore-arc basin clastic rocks (Cretaceous, south Tibet). Geol. Soc. Am. Bull. 108, 669-684.

Elthon, D. (1991) Geochemical evidence for formation of Bay of Islands ophiolite above a subduction zone. Nature 354, 140-143.

Girardeau, J. and Mercier, J. C. (1988) Petrology and texture of the ultramafic rocks of the Xigaze ophiolite (Tibet): constraints for mantle structure beneath slowspreading ridges. Tectonophys. 47, 33-58.

Girardeau, J., Mercier, J. C. C. and Cao, Y. G. (1985) Origin of the Xigaze ophiolite, Yarlung Zangbo Suture Zone, Southern Tibet. Tectonophys. 119, 407433.

Gopel, C., Allegre, C. J. and Xu, R. H. (1984) Lead isotope study of the Xigaze ophiolite (Tibet): the problem of the relationship between magmatites (gabbros, dolerites, lavas) and tectonites (harzburgites). Earth Planet. Sci. Lett. 69, 301-310.

Govindaraju, K. (1994) 1994 compilation of working values and sample description for 383 geostandards. Geostand. Newsletter 18, 158 pp.

Gruau, G., Bernard-Griffiths, J. and Lecuyer, C. (1998) The origin of U-shaped rare earth patterns in ophiolite peridotites: Assessing the role of secondary alteration and melt/rock reaction. Geochim. Cosmochim. Acta 62, 3545-3560.

Hao, J., Chai, Y. C. and Li, J. L. (1999) Original tectonic setting of the Tsangpo ophiolite and sedimentary evolution of the Xigaze forearc basin. Sci. Geol. Sin. 34, 1-9.

Jenner, G. A., Dunning, G. R., Malpas, J., Brown, M. and Brace, T. (1991) Bay of Islands and Little Port complexes, revisited: age, geochemistry and isotopic evidence confirm suprasubduction-zone origin. Can.
Jour. Ear. Sci. 28, 1635-1652.

Marcoux, J. and 9 others (1982) Preliminary report on depositional sediments on top of the volcanic member: the Xigaze ophiolite (Yarlung-Zangbo suture zone). Ofioliti 2/3, 395-396.

Meschede, M. (1986) A method of discriminating between different types of mid-ocean ridge basalts and continental tholeiites with the $\mathrm{Nb}-\mathrm{Zr}-\mathrm{Y}$ diagram. Chem. Geol. 56, 207-218.

Mullen, E. D. (1983) $\mathrm{MnO} / \mathrm{TiO}_{2} / \mathrm{P}_{2} \mathrm{O}_{5}$ : a minor element discriminant for basaltic rocks of oceanic environments and its applications for petrogenesis. Earth Planet. Sci. Lett. 62, 53-62.

Nicolas, A. and 6 others (1981) The Xigaze ophiolite: a peculiar oceanic lithosphere. Nature 294, 414-417.

Pearce, J. A. (1975) Basalt geochemistry used to investigate past tectonic environments on Cyprus. Tectonophys. 25, 41-67.

Pearce, J. A. (1982) Trace element characteristics of lavas from destructive plate boundaries. Andesites: Orogenic Andesites and Related Rocks (Thorpe, R. S., ed.), 525-554, John Wiley \& Sons, New York.

Pearce, J. A. (1991) Ocean floor comes ashore. Nature 354, 110-111.

Pearce, J. A. and Cann, J. R. (1973) Tectonic setting of basic volcanic rocks determined using trace element analyses. Earth Planet. Sci. Lett. 19, 290-300.

Pearce, J. A. and Deng, W. M. (1988) The ophiolites of the Tibet Geotraverses, Lhasa to Golmud (1985) and Lhasa to Kathmandu (1986). Phil. Trans. R. Soc. Lond. 327, 215-238.

Pearce, J. A. and Norry, M. J. (1979) Petrogenetic Implications of $\mathrm{Ti}, \mathrm{Zr}, \mathrm{Y}$, and $\mathrm{Nb}$ variations in volcanic rocks. Contrib. Mineral. Petrol. 69, 33-47.

Pearce, J. A., Lippard, S. J. and Roberts, S. (1984) Characteristics and tectonic significance of suprasubduction zone ophiolites. Marginal Basin Geology (Kokelaar, B. P. and Howell, M. F., eds.), Geol. Soc. Lond. Spec. Pub. 16, 77-93.

Qi, L., Hu, J. and Gregoire, D. C. (2000) Determination of trace elements in granites by inductively coupled plasma mass spectrometry. Talanta 51, 507-513.

Shervais, J. W. (1982) Ti-V plots and the petrogenesis of modern and ophiolitic lavas. Earth Planet. Sci. Lett. 59, 101-118.

Suen, C. J., Frey, F. A. and Malpas, J. (1979) Bay of Islands ophiolites suite, Newfoundland: Petrologic and geochemical characteristics with emphasis on rare earth element geochemistry. Earth Planet. Sci. Lett. 45, 337-348.

Sun, S. S. (1982) Chemical composition and origin of the Earth's primitive mantle. Geochim. Cosmochim. Acta 46, 179-192.

Sun, S. S., Nesbitt, R. W. and Sharaskin, A. Y. (1979) Geochemical characteristics of mid-ocean ridge 
basalts. Earth Planet. Sci. Lett. 44, 119-138.

Tapponnier, P. and 34 others (1981) The Tibetan side of the India-Eurasia collision. Nature 294, 405-410.

Taylor, S. R. and McLennan, S. M. (1985) The Continental Crust: Composition and Evolution. Blackwell Scientific Publications, 54, 209-372.

Thy, P. and Moores, E. M. (1988) Crustal accretion and tectonic setting of the Troodos ophiolite, Cyprus. Tectonophys. 147, 221-245.

Wang, C. S., Liu, Z. F. and He, Z. W. (1999) Speculations on the early Cretaceous Yarlung Zangbo Palaeoophiolite in Southern Tibet. Acta Geol. Sin. 73, 714.

Wang, X. B., Bao, P. S. and Xiao, X. C. (1987) Ophiolites of the Yarlung Zangbo River (Tibet). Geol. Pub. House, Beijing, 118 pp.

Wood, D. A. (1980) The application of a Th-Hf-Ta diagram to problems of tectonomagmatic classification and to establishing the nature of crustal contamination of basaltic lavas of the British Tertiary volcanic province. Earth Planet. Sci. Lett. 50, 11-30.

Wu, H. R. (1984) The Congdui Formation-Cretaceous deep-sea deposits in southern Xizang (Tibet) and its significance. Sci. Geol. Sin. 1, 26-33.

XZBGM (Xizang Bureau of Geology and Mineral Resources) (1993) Regional Geology of Xizang (Tibet) Autonomous Region. Geol. Pub. House, Beijing, 264477.

Zhang, K. J. (2000) Cretaceous paleogeography of Tibet and adjacent areas (western China): tectonic implications. Cret. Res. 21, 23-33.

Zhou, M.-F., Robinson, P. T., Malpas, J. and Li, Z. (1996) Podiform chromitites from the Luobusa ophiolite (Southern Tibet): Implications for meltmantle interaction and chromite segregation in the upper mantle. J. Petrol. 37, 3-21.

Zhou, M.-F., Yumul, G. P., Jr., Malpas, J. and Sun, M. (2000) Comparative study of platinum-group elements in the Acoje and Coto Blocks of the Zambales ophiolite, Philippines. The Island Arc 9, 556-564.

Zhou, M.-F., Robinson, P. T., Malpas, J., Aitchison, J., Sun, M., Bai, W.-J., Hu, X.-F. and Yang, J.-S. (2001) Melt/mantle interaction and melt evolution in the Sartohay high-Al chromite deposit of the Dalabute ophiolite (NW China). J. Asian Earth Sci. 19, $517-$ 534. 